\title{
Emotional states as distinct configurations of functional brain networks
}

Rotem Dan ${ }^{1,2}$, Marta Weinstock ${ }^{3}$, Gadi Goelman ${ }^{2}$

${ }^{1}$ Edmond and Lily Safra Center for Brain Sciences (ELSC), The Hebrew University of Jerusalem, Jerusalem, Israel

${ }^{2}$ Department of Neurology, Hadassah Hebrew University Medical Center, Jerusalem, Israel

${ }^{3}$ Institute of Drug Research, The Hebrew University of Jerusalem, Jerusalem, Israel

Correspondence to:

Rotem Dan

Center for Depression, Anxiety and Stress Research, McLean Hospital

Department of Psychiatry, Harvard Medical School

115 Mill St, Belmont, MA 02478, USA

Phone: +16178552919, Email: rdanyogev@ mclean.harvard.edu

Abbreviated title: Brain connectome of emotion 


\begin{abstract}
The conceptualization of emotional states as patterns of interactions between large-scale brain networks has recently gained support. Yet, few studies have directly examined the brain's network structure during emotional experiences. Here, we investigated the brain's functional network organization during experiences of sadness, amusement, and neutral states elicited by movies, in addition to a resting-state. We tested the effects of the experienced emotion on individual variability in the brain's functional connectome. Next, for each state, we defined a community structure of the brain and quantified its segregation and integration. Our results show that emotional states increase the similarity between and within individuals in the brain's functional connectome. Second, in the brain's modular organization, sadness, relative to amusement, was associated with higher integration and increased connectivity of cognitive control networks: the salience and fronto-parietal networks. Importantly, the modular metrics of brain segregation and integration were related to the subjective emotional valence. Last, in both the functional connectome and emotional report, we found higher similarity among women. Our results suggest that the experience of emotion is linked to a reconfiguration of whole-brain distributed, not emotion-specific, functional brain networks and that the brain's topological structure carries information about the subjective emotional experience.
\end{abstract}

Keywords: Emotion; Connectome; fMRI; Modularity; Movie 


\section{Introduction}

The encoding of emotion in the human brain remains an area of ongoing debate. Traditionally, most studies sought to map specific categories of emotion to localized brain regions or anatomical circuits, focusing on the role of subcortical structures ${ }^{1,2}$. However, it has become increasingly evident that there is no one-toone mapping between discrete emotions and individual brain regions ${ }^{3}$. With the evolution of cognitive network neuroscience, the study of the neural basis of emotion has shifted to the examination of functional brain networks ${ }^{4}$. The recent application of machine learning techniques to neuroimaging data demonstrated that specific categories of emotion can be distinguished based on neural activity patterns, distributed over the cortex and subcortex ${ }^{5,6}$.

This new line of research may suggest that there is no brain region or even a brain network, that is specific to a certain emotion. Rather, emotional states are thought to result from patterns of interactions between multiple large-scale brain networks ${ }^{4,6,7}$. These functional brain networks are not only involved in emotion but also in non-emotional processes (such as conceptualization, attention, motor function, and executive control), and are not dedicated to an emotion category, but instead, their specific interactions are postulated to be the basis for an emotional state. However, surprisingly, despite much theoretical interest, few studies have directly examined the brain's network structure during subjective experiences of emotion. Thus, following these experimental gaps, this study's goals were twofold. First, to delineate the manner in which emotional states influence individual differences in brain functional connectivity. Second, defining the community structure of the brain during specific emotional experiences.

Outside the field of emotion, the effects of the task or mental state on individual differences in the brain's functional connectome have recently gained interest ${ }^{8,9}$. A functional connectome refers to the communication patterns between brain regions, typically computed by the level of synchronization between fMRI blood-oxygen-level-dependent (BOLD) signals ${ }^{10}$. The effects of the mental state on variability in the functional connectome can be evaluated by examining: (i) within-subject similarity, i.e., how functional connectomes acquired during different states are similar within an individual, and (ii) between-subject similarity, how functional connectomes acquired during a specific state are similar across individuals. 
Previous studies, such as that of Finn and colleagues ${ }^{11}$, showed that both the within- and between-subject similarities are dependent on the state.

The mental state was further shown to influence the brain's modular organization. As a complex system, the brain can be divided into communities of regions, called modules, that are functionally highly intra-connected and less strongly functionally inter-connected ${ }^{12}$. Describing the brain from a network science perspective allows one to quantify higher-level complex interactions between the network's elements (e.g. brain regions), and examine their link to the mental state and behavior ${ }^{13}$. For example, Cohen and D'Esposito ${ }^{14}$ found increased modular segregation during a motor task as opposed to enhanced global integration during a working memory task.

Crucially, disturbances of emotion are key components in many psychiatric disorders, such as depression, anxiety, and autism. Understanding how emotional states are encoded in the brain's complex functional structure, not only by the macro-scale networks that are involved but also by the specific communication patterns between them, and how they are dependent on the subjective emotional experience, could be critical for developing brain-based models that can predict disease trajectory and clinical outcome, ultimately advancing the clinical utility of neuroimaging methods.

In the current study, we aimed to: (i) Examine the effects of the emotional experience and sex on the within- and between-subject similarity in the brain's functional connectome. We hypothesized that the emotional state would increase the similarity in the brain's global organization, both between individuals and between states within individuals. Given the well-established sex differences in mood disorders ${ }^{15}$, we hypothesized that sex would interact with the emotional state. (ii) Define the modular organization of the brain during specific emotions: sadness and amusement. These emotional categories were chosen based on their relevance to clinical disorders, such as depression and bipolar disorder ${ }^{16-18}$, and their opposed emotional valence. (iii) Quantify differences between emotional states in the segregation and integration of network communities. In line with the notion of emotional states as distinct interactions between largescale functional brain networks, we postulated that sadness and amusement, despite being both high-order 
complex cognitive states, will differ in their level of modular segregation and in specific patterns of between-module communication.

\section{Methods}

\section{Participants}

Healthy young participants were recruited among undergraduate students at the Hebrew University of Jerusalem. Exclusion criteria included: past or present psychiatric or neurological disorders; use of psychiatric medication. One participant was excluded due to anxiety in the MRI scanner, and another was excluded based on in-scanner motion (Supplement). The final analyzed sample was composed of 50 healthy participants ( 30 women, 20 men; age: $23.94 \pm 2.64$ years). The study was approved by the Hadassah Hebrew University Medical Center Ethics Committee and was carried out in compliance with the Declaration of Helsinki.

\section{Emotional brain states using movies}

Each fMRI scan included the following sequence to induce four different mental states: (i) restingstate (10 minutes): eyes open, fixating on a visual crosshair; (ii, iii) two emotional states (10 minutes each): sadness and amusement, induced by continuous exposures to movies, and (iv) a neutral movie state (10 minutes). Each movie state (sadness, amusement, and neutral) was induced by four movie clips (2-3 minutes each) presented in a row. The order of the emotional states (sadness, amusement) was counterbalanced across participants with the neutral state between them. We chose movies since we wanted to probe emotion in a naturalistic way, including an intense emotional experience. Movie-watching is a powerful naturalistic method to induce engaging and rich high-order cognitive states in the MRI scanner ${ }^{19}$. With regards to brainbehavior relations, movie-watching was shown to increase the accuracy of predicting cognitive traits from brain functional data ${ }^{20}$. Another advantage of movie-watching is that it commonly reduces in-scanner head motion and thus can improve data quality ${ }^{21}$. 
Participants rated after the scan their subjective emotional experience during each movie state for 18 discrete emotional categories, valence (i.e., the affective quality of the experience, good/positive vs. bad/negative) and arousal, on a 1-to-8 Likert scale, using a similar questionnaire to that of Gross and colleagues ${ }^{22}$. The movies were taken from available sets ${ }^{22-25}$, tested in a separate behavioral study on an independent group of participants, and chosen such that they induce the targeted discrete emotion (i.e., sadness and not a mixture of sadness and fear) with a high level of intensity. Detailed information regarding the movie clips and the behavioral testing procedure was previously reported ${ }^{26}$. The movies were matched for the duration, number of actors, and social interaction. All movies were in English with Hebrew subtitles.

\section{MRI data acquisition and preprocessing}

MRI data were acquired on a 3T Magnetom Skyra. Preprocessing of fMRI data was done using SPM12. Details of acquisition, preprocessing and in-scanner motion correction are found in the Supplement.

\section{Functional connectome for each brain state}

For each participant and mental state (i.e., sadness, amusement, neutral, rest), a whole-brain functional connectome covering the cortex and subcortex (excluding the cerebellum) was computed using CONN ${ }^{27}$. The brain was parceled to nodes and the average blood-oxygenation-level-dependent (BOLD) time series was calculated for each node. Pearson's correlations were computed between time series from all pairs of nodes and Fisher's transform was applied to correlation values, resulting in symmetric connectivity matrices.

In network analysis of the brain, the effect of the atlas used for node definition is an area of an ongoing investigation. We, therefore, calculated functional connectomes using four different atlases: (i) Automated anatomical labeling atlas (AAL) ${ }^{28}$ : 90 nodes; (ii) Harvard-Oxford atlas (HO) ${ }^{29}$ : 105 nodes; (iii) Shen atlas ${ }^{30}: 218$ nodes (excluding the cerebellum); (iv) Schaefer atlas ${ }^{31}: 400$ nodes. The brain stem and cerebellum were excluded from the atlases. Since the Schaefer brain subdivision does not include the subcortex, 14 subcortical nodes from the Harvard-Oxford atlas were added to this atlas, resulting in a total 
of 414 nodes. Note that the AAL and Harvard-Oxford subdivisions were defined based on anatomical features whereas Shen and Schaefer defined parcels based on the functional homogeneity of resting-state time series.

\section{Functional connectome similarity analysis}

For each parcellation atlas, functional connectivity matrices were thresholded at $\mathrm{z}=0$ (i.e., negative weights were set to zero) to include only positive correlations (see Supplement for analysis that included negative correlations). To examine within- and between-subject similarity in the functional connectome, we followed previous approaches ${ }^{11,32}$ : the unique elements of each connectivity matrix were extracted by taking the upper triangle of the matrix, resulting in a vector of edge values for each participant for each mental state. The between-subject similarity was calculated using Pearson's correlation between all possible pairs of participants for a certain state. These values were Fisher's z-transformed, averaged across participants, and reverted to $r$ values, resulting in a single value per participant. The within-subject similarity was calculated using Pearson's correlation between all pairs of states for each participant, resulting in six values per participant (sadness vs. amusement, sadness vs. neutral, amusement vs. neutral, sadness vs. rest, amusement vs. rest, neutral vs. rest).

To examine the effect of sex on between-subject similarity, we calculated similarity among women (i.e., between each woman and all other women) and among men, denoted here as women-to-women and men-to-men similarity. Statistical analyses were conducted with SPSS v.23 (SPSS Inc., Chicago, IL) on the Fisher transformed z-values. Differences in within- and between-subject similarity were tested by 2-way mixed ANOVA models implemented by a GLM with state as a within-subject factor and sex as a betweensubject factor. Post-hoc pairwise comparisons were conducted using Sidak correction. 


\section{Modularity analysis}

\section{Community detection for each brain state}

To decompose the entire brain network into functional communities for each mental state, modularity analysis was conducted on the weighted functional connectivity matrices using a consensus partitioning algorithm ${ }^{33}$ implemented in the Brain Connectivity Toolbox (BCT) ${ }^{34}$. This algorithm partitions a wholebrain network into nonoverlapping groups of brain regions, i.e., "communities" or "modules", by maximizing a modularity function. The Harvard-Oxford atlas was chosen for node definition since it showed greater between-subject similarity in the functional connectome per state, compared to the higher resolution network parcellations of Shen and Schaefer (see Results and Figure 2a). In the first step, for each participant and each state separately, we applied a Louvain modularity optimization procedure 500 times to define an "agreement" matrix. Each cell in the agreement matrix corresponded to the proportion of times that a pair of nodes were assigned to the same community over the 500 repetitions. At the next step, Louvain modularity optimization was conducted on the agreement matrix, with 500 repetitions, generating a new agreement matrix. This step was repeated until convergence, i.e., until the agreement matrix was deterministic: containing only zeros or ones.

After a consensus partition was identified for each subject, we computed a group-level consensus partitioning for each state. The consensus partitions of all subjects for a specific state were combined to create a group-level agreement matrix and Louvain modularity optimization was conducted on the agreement matrix until convergence. This procedure yielded a community structure, or modular organization, for each state at the individual and group levels. The consensus partitioning procedure was repeated over a range of the resolution parameter gamma ${ }^{35}$ and with different treatments of negative weights in the modularity function ${ }^{36}$. A resolution parameter gamma=2.25 and a positive-only weighted modularity function were chosen. Details on the choice of the resolution parameter and modularity function are found in the Supplement.

To compute the similarity within- and between-participants in community structure, we used the normalized mutual information (NMI) ${ }^{37}$. The NMI measures the dependency between two node 
assignments, i.e., how much information one set of assignments provides about a second set of assignments and ranges from 0 (no information/similarity) to 1 (identical community structure).

\section{Modular segregation and integration metrics}

After each node was assigned to a group-level module for each mental state, we calculated the following measures of modular segregation and integration. Note that all measures retain the weights of the functional connections and were calculated for a specific state.

(i) System segregation ${ }^{38}$ : the normalized difference between within- and between-module connectivity. A higher value indicates greater segregation, i.e., less interaction between modules. This measure was calculated for each module, resulting in a modular segregation metric as follows:

$$
\text { System segregation }_{i}=\frac{\overline{\mathrm{Z}}_{\mathrm{w}, \mathrm{i}}-\overline{\mathrm{Z}}_{\mathrm{b}, \mathrm{i}}}{\overline{\mathrm{Z}}_{\mathrm{w}, \mathrm{i}}}
$$

where $\bar{Z}_{\mathrm{w}, \mathrm{i}}$ is the mean functional connectivity (Fisher transformed z-values) between all pairs of nodes within module $\mathrm{i}$ and $\overline{\mathrm{Z}}_{\mathrm{b}, \mathrm{i}}$ is the mean functional connectivity between nodes of module $\mathrm{i}$ and nodes of all other modules.

(ii) Participation coefficient ${ }^{39}$ : quantifies the diversity of a node's connections across modules. It is close to 1 if the node's connections are distributed uniformly among all modules and 0 if all connections are within the node's module. A high participation coefficient indicates high integration, i.e., strong connections to many modules, whereas a low participation coefficient indicates high segregation, i.e., weak interactions with other modules. For each module, we calculated the mean participation coefficient across all its nodes. The weighted variant of the participation coefficient from the BCT was used:

$$
\text { Participation coefficient }_{i}=1-\sum_{\mathrm{m} \in \mathrm{M}}\left(\frac{\mathrm{k}_{\mathrm{i}}(\mathrm{m})}{\mathrm{k}_{\mathrm{i}}}\right)^{2}
$$

where $M$ is the set of modules $m, k_{i}(m)$ is the weighted degree (i.e., summed weighted functional connectivity) of node $\mathrm{i}$ to nodes within module $m$ and $k_{i}$ is the total weighted connectivity of node $\mathrm{i}$ to all nodes regardless of module membership. 
(iii) Pairwise between-module connectivity: defined as the mean functional connectivity strength (Fisher transformed z-values) between pairs of modules, averaged across all possible connections. For example, the connectivity between module $\mathrm{i}$ and module $\mathrm{j}$ was calculated as the sum of functional connections between nodes in module $\mathrm{i}$ and nodes in module $\mathrm{j}$, divided by the number of possible connections (number of nodes in module $\mathrm{i} x$ number of nodes in module $\mathrm{j}$ ).

To examine differences between the four mental states in overall modular segregation and integration, the mean system segregation (across all modules), mean participation coefficient (across all nodes), and the modularity quality index (Q) (i.e., the modularity function that was maximized during the consensus clustering) were computed and analyzed by 1-way ANOVA models. A higher value of Q indicates a more modular organization. The overall functional connectivity strength (including positive connections only) was also calculated for each mental state and analyzed by 1-way ANOVA models.

Statistical differences between the emotional states (sadness, amusement) in segregation and integration of specific modules, were tested for the modules that were common to both emotions by 2-way repeated-measures ANOVA models implemented by a GLM with emotion and module as within-subject factors. Post-hoc pairwise comparisons were conducted using Sidak correction. In addition, for each emotional state, the association between segregation and integration metrics and the reported valence of the emotional experience was computed using Pearson's correlation, and correlation values were FDR corrected for multiple comparisons at $p<0.05$.

\section{Behavioral report of emotion experience}

Valence, arousal, and interest were rated for the three movie states: sadness, amusement, neutral. The intensity of the target emotion was calculated only for the emotional states: sadness and amusement. The intensity of sadness was calculated as the average rating for "sadness" and "sorrow" and the intensity of amusement was calculated as the average of "amusement" and "pleasure". Arousal was added to the postfilm questionnaire after the beginning of the study; thus, ratings were missing from 9 participants for all states. The rating of valence was missed for amusement from one participant. 
The between-subject similarity in the behavioral report of emotion was computed for each movie state (sadness, amusement, neutral), utilizing a similar approach to the one used for the functional connectome. The vector of ratings for the 19 items (emotional categories, valence) was extracted for each participant for each state. The between-subject similarity was calculated using Pearson's correlation between all possible pairs of participants for a certain state. These values were Fisher's z-transformed and averaged across participants resulting in a single value per participant. To examine the effect of sex, we calculated similarity among women and men, i.e., women-to-women and men-to-men similarity.

For each behavioral measure (valence, intensity, arousal, interest, between-subject similarity), statistical analysis was conducted in SPSS on the Fisher transformed z-values by 2-way mixed ANOVA models implemented by a GLM with state as a within-subject factor and sex as a between-subject factor. Post-hoc pairwise comparisons were conducted using Sidak correction.

\section{Results}

\section{Within-subject similarity in the brain's functional connectome}

The within-subject similarity was dependent on the state [main effect of state, all $p<10^{-6}$, GreenhouseGeisser corrected: AAL: $\quad F(3.58,172.25)=102.50$, partial eta-squared: $\quad \eta_{\mathrm{p}}{ }^{2}=0.68 ; \quad \mathrm{HO}$ : $F(3.73,179.25)=129.83, \quad \eta_{\mathrm{p}}{ }^{2}=0.73 ; \quad$ Shen: $\quad F(3.62,173.99)=177.27, \quad \eta_{\mathrm{p}}{ }^{2}=0.78 ; \quad$ Schaefer: $\left.F(3.56,171.15)=200.71, \eta_{\mathrm{p}}^{2}=0.80\right]$.

An individual's functional connectome was more similar between two movie states (sadness, amusement, neutral) than between a movie state and rest, regardless of the brain parcellation atlas (pairwise differences: Cohen's $d=1.35-3.08$ ) (Figure 1). Furthermore, for all atlases except for the AAL, the functional connectome was more similar between two emotional states (sadness, amusement) than between an emotional state and the neutral movie state (Cohen's $d=0.43-0.81$ ). There was no effect of sex or sexby-state interaction (Supplemental Figure S6). 


\section{Between-subject similarity in the brain's functional connectome}

Parcellating the brain into more nodes resulted in an overall decrease in similarity between participants (number of parcels: Schaefer $>$ Shen $>\mathrm{HO}>\mathrm{AAL}$ ) (Figure 2). The between-subject similarity was dependent on the state [main effect of state, all $p<10^{-6}$, Greenhouse-Geisser corrected: AAL: $F(2.17,76.106 .55)=292.72, \quad \eta_{\mathrm{p}}{ }^{2}=0.85 ; \quad$ HO: $\quad F(2.26,111.04)=1263.05, \quad \eta_{\mathrm{p}}{ }^{2}=0.96 ; \quad$ Shen: $F(2.21,108.63)=470.38, \eta_{\mathrm{p}}{ }^{2}=0.90$; Schaefer: $\left.F(1.68,82.60)=55.97, \eta_{\mathrm{p}}{ }^{2}=0.53\right]$ (Figure 2a).

Participants were more similar to one another during emotional states (sadness, amusement) than during the neutral movie state, regardless of the parcellation atlas [(i) sadness vs. neutral: Cohen's $d=4.72$, 9.55, 5.32, 2.69 for AAL, HO, Shen and Schaefer atlases respectively; (ii) amusement vs. neutral: Cohen's $d=1.79,3.69,5.02$ for AAL, HO and Shen atlases respectively, not significant for Schaefer: $d=0.34]$. Furthermore, higher between-subject similarity was found during sadness relative to amusement [Cohen's $d=2.34,3.62,1.06$ for AAL, HO, and Schaefer atlases respectively]. The Shen atlas showed an opposite and smaller effect (Cohen's $d=0.55)$.

Women were more similar to other women than men to other men, across states, for all atlases except for the Shen atlas [main effect of sex: AAL: $F(1,48)=15.21, p=2.9 \cdot 10^{-4}, \eta_{\mathrm{p}}{ }^{2}=0.24 ;$ HO: $F(1,48)=22.80, p=1.7 \cdot 10^{-5}, \eta_{\mathrm{p}}{ }^{2}=0.32 ;$ Schaefer: $\left.F(1,48)=20.94, p=3.4 \cdot 10^{-5}, \eta_{\mathrm{p}}{ }^{2}=0.30\right]$. Sex differences varied by state [sex-by-state interaction: AAL: $F(1.59,76.30)=37.03, \quad p<10^{-6}, \quad \eta_{\mathrm{p}}{ }^{2}=0.43 ; \quad \mathrm{HO}$ : $F(1.55,74.50)=32.17, \quad p^{<10^{-6}}, \quad \eta_{\mathrm{p}}^{2}=0.40 ; \quad$ Shen: $\quad F(1.61,77.29)=15.67, \quad p=1 \cdot 10^{-5}, \quad \eta_{\mathrm{p}}^{2}=0.24 ; \quad$ Schaefer: $F(2.05,98.68)=19.98, p^{<10^{-6}}, \eta_{\mathrm{p}}{ }^{2}=0.29$; all Greenhouse-Geisser corrected] (Figure 2b). Amusement showed significant sex differences across all atlases (Cohen's $d=0.44-0.96)$.

\section{Behavioral report of emotional experience}

\section{Valence, intensity, arousal, and interest}

\section{(i) Valence}

A significant main effect of state was found, as expected: amusement $>$ neutral $>$ sadness $[F(2,94)=53.46$, $p<10^{-6}, \quad \eta_{\mathrm{p}}{ }^{2}=0.53$. Ratings $\quad$ on 1 -to- 8 Likert scale: amusement $=6.77 \pm 1.10 ;$ neutral $=5.36 \pm 1.36$; 
sadness $=4.28 \pm 1.79]$. Namely, emotional experience during induction of amusement was rated as "pleasant/positive" whereas the experience during induction of sadness was rated as "unpleasant/negative". There were no sex or interaction effects.

(ii) Arousal

A significant main effect of state was found: higher arousal for emotional states relative to neutral, without a difference between emotional states $\left[F(1.63,63.75)=47.45, \quad p<10^{-6}, \quad \eta_{\mathrm{p}}{ }^{2}=0.54\right.$, Greenhouse-Geisser corrected. Ratings on 1-to- 8 Likert scale: amusement=6.04 \pm 1.39 ; sadness $=5.63 \pm 1.75$; neutral=3.29 \pm 1.74$]$. There were no sex or interaction effects.

(iii) Intensity of target emotion

Emotional states (sadness, amusement) did not differ in the reported intensity of target emotion, and there was no effect of sex [Ratings on 1-to- 8 Likert scale: amusement $=6.15 \pm 1.34$; sadness $=5.89 \pm 1.54]$.

(iv) Interest

Higher interest was reported for amusement relative to sadness and for both emotional states relative to neutral: amusement $>$ sadness $>$ neutral [main effect of state: $F(2,96)=47.10, p<10^{-6}, \eta_{p}^{2}=0.49$. Ratings on 1 to-8 Likert scale: amusement $=5.78 \pm 1.65$; sadness $=5.02 \pm 1.93$; neutral $=2.88 \pm 1.57]$. There were no sex or interaction effects.

\section{Between-subject similarity in the behavioral report}

Participants were more similar to one another in their reports of emotional experience during amusement, relative to sadness or the neutral state [main effect of state: $F(2,98)=2774.61, p<10^{-6}, \eta_{\mathrm{p}}{ }^{2}=0.98$ ] (Figure 3a). In addition, women were more similar to other women than men to other men [main effect of sex: $F(1,48)=17.20, p=1.36 \cdot 10^{-4}, \eta_{\mathrm{p}}{ }^{2}=0.26$. main effect of emotion: $\left.F(2,96)=1236.42, p<10^{-6}, \eta_{\mathrm{p}}{ }^{2}=0.96\right]$ (Figure 3b). There was no sex-by-emotion interaction. 


\section{Modular organization of different brain states}

The community structure identified for sadness, amusement, neutral, and rest is presented in Figure 4 (see Supplemental Table S1 for the list of node assignments to modules). Each brain state was characterized by a specific topological organization. Eight modules were identified for amusement, neutral, and rest, and nine for sadness. Several qualitative differences in the community structure were observed between the four brain states. The salience network was identified as a separate community only in sadness and amusement. For sadness and neutral, the occipital cortex was divided into two networks: primary and secondary visual, as opposed to one visual network in amusement and rest. Separate sensorimotor and auditory communities were identified in sadness and amusement, whereas in neutral and rest these networks were combined into one community. The posterior default mode network emerged as a separate community only in the resting state.

For the modularity quality index (Q), a main effect of the mental state was found $\left[F(2.48,121.89)=5.25, p=0.004, \eta_{\mathrm{p}}^{2}=0.09\right.$, Greenhouse-Geisser corrected], with higher $\mathrm{Q}$ for amusement relative to rest ( $p=0.002$, Cohen's $d=0.55$ ), without a significant difference between the emotional states ( $p=0.055$, Cohen's $d=0.38$ ). A main effect of the mental state was also found for the overall functional connectivity strength (positive connections only) $\left[F(3,147)=6.43, p=4 \cdot 10^{-4}, \eta_{\mathrm{p}}{ }^{2}=0.11\right]$, with higher overall connectivity indicated for rest relative to amusement and sadness (rest>amusement: $p=0.001$, Cohen's $d=0.60$; rest>sadness: $p=0.008$, Cohen's $d=0.48$, without a difference between the emotional states $(p=0.52$, Cohen's $d=0.09)$.

\section{Within- and between-subject similarity in the brain's community structure}

The within-subject similarity in the community structure was dependent on the state $\left[F(3.37,165.38)=22.12, p^{<10^{-6}}, \eta_{\mathrm{p}}{ }^{2}=0.31\right.$, Greenhouse-Geisser corrected] (Supplemental Figure S10). An individual's community structure was more similar between two movie states (sadness, amusement, neutral) than between a movie state and rest (Cohen's $d=0.53-1.05)$. Also, greater similarity was found 
between the two emotional states (sadness, amusement) than between amusement and the neutral movie state (Cohen's $d=0.46)$.

The between-subject similarity in the community structure was dependent on the state $\left[F(3,147)=7.96, p=5.9 \cdot 10^{-5}, \eta_{\mathrm{p}}{ }^{2}=0.14\right]$ (Supplemental Figure S11). Individuals were more similar to one another during sadness, compared to all other states (Cohen's $d=0.61,0.43,0.64$ for amusement, neutral, and rest respectively).

\section{Modular segregation and integration during emotional brain states}

The mean system segregation (across all modules) and the overall mean participation coefficient (across all nodes) were higher for sadness than amusement and for both emotional states than rest [main effect of state: (i) system segregation: $\quad F(3,147)=13.38, \quad p<10^{-6}, \quad \eta_{\mathrm{p}}^{2}=0.21$; (ii) participation coefficient: $F(2.49,122.10)=54.23, p<10^{-6}, \eta_{\mathrm{p}}{ }^{2}=0.52$, Greenhouse-Geisser corrected] (Figures 5a,b). To compare the segregation and integration of specific modules between the emotional states, further analysis was conducted on the seven modules that were common to both emotions: (1) fronto-parietal; (2) salience; (3) language; (4) sensorimotor; (5) auditory; (6) limbic; and (7) basal ganglia. Visual modules were excluded since they were not comparable between the emotional states (two modules identified in sadness and one in amusement).

For the system segregation, a main effect of module was found $\left[F(4.92,241.11)=22.29, p<10^{-6}\right.$, $\eta_{\mathrm{p}}^{2}=0.31$, Greenhouse-Geisser corrected] (Figure 5c). The highest modular segregation was indicated for the auditory module and the lowest segregation for the basal ganglia module. A module-by-emotion interaction was also found $\left[F(4.58,224.64)=39.50, p<10^{-6}, \eta_{\mathrm{p}}^{2}=0.44\right.$, Greenhouse-Geisser corrected]: in sadness relative to amusement, higher segregation was indicated for the salience and auditory modules whereas in amusement higher segregation was indicated for the fronto-parietal and language networks.

For the participation coefficient, main effects of emotion, module and emotion-by-module interaction were found [emotion: $F(1,49)=21.83, p=2.4 \cdot 10^{-5}, \eta_{\mathrm{p}}{ }^{2}=0.30$; module: $F(4.61,226.13)=65.22$, $p<10^{-6}, \eta_{\mathrm{p}}^{2}=0.57$, Greenhouse-Geisser corrected; emotion-by-module: $F(4.69,229.87)=97.37, p<10^{-6}$, 
$\eta_{\mathrm{p}}{ }^{2}=0.66$, Greenhouse-Geisser corrected] (Figure 5d). Across modules, overall greater diversity of connections, i.e., integration, was indicated in sadness. Furthermore, for both emotions, the highest diversity of connections was indicated for the basal ganglia module. In sadness relative to amusement, higher integration was indicated for the fronto-parietal, salience, language, limbic and basal ganglia modules whereas in amusement higher integration was indicated for the auditory module.

Specific between-module connections were further examined with the pairwise between-module connectivity. Main effects of emotion, connection and emotion-by-connection interaction were found [emotion: $F(1,49)=4.27, \quad p=0.044, \quad \eta_{\mathrm{p}}^{2}=0.08 ;$ connection: $\quad F(8.99,440.62)=97.39, \quad p<10^{-6}, \quad \eta_{\mathrm{p}}^{2}=0.66$, Greenhouse-Geisser corrected; emotion-by-connection: $\quad F(9.62,471.53)=7.49, \quad p<10^{-6}, \quad \eta_{\mathrm{p}}^{2}=0.13$, Greenhouse-Geisser corrected] (Figure 5e). Overall greater between-module connectivity was found for sadness, as indicated above by the participation coefficient. In sadness compared to amusement, higher between-module connectivity was found for the frontoparietal-salience, frontoparietal-language, frontoparietal-limbic, frontoparietal-auditory, salience-language, salience-sensorimotor, salience- limbic, salience-basal ganglia, limbic-language, and language-auditory connectivity. In amusement relative to sadness, higher between-module connectivity was found for the frontoparietal-sensorimotor and sensorimotor-auditory connections.

\section{Relationship between modular metrics and reported emotional valence}

The system segregation of the fronto-parietal and salience modules was associated with the reported emotional valence [fronto-parietal: $p=1.41 \cdot 10^{-4}, \mathrm{r}=0.41$; salience: $p=4.26 \cdot 10^{-4}, \mathrm{r}=-0.37$; all FDR corrected], in line with the higher segregation of the fronto-parietal module in amusement and higher segregation of the salience module in sadness.

The mean participation coefficients of the fronto-parietal, salience, language, limbic and auditory modules were associated with the reported valence [fronto-parietal: $p=2.1 \cdot 10^{-5}, \mathrm{r}=-0.44$; salience: $p=0.003$, $\mathrm{r}=-0.30$; language: $p=2.45 \cdot 10^{-4}, \mathrm{r}=-0.38$; limbic: $p=0.001, \mathrm{r}=-0.32$; auditory: $p=5.01 \cdot 10^{-4}, \mathrm{r}=0.36$; all FDR 
corrected], in line with the higher participation coefficients found in sadness, except for the auditory module.

The pairwise between-module connectivity was associated with the reported valence [frontoparietal-limbic: $p=1.09 \cdot 10^{-4}, \mathrm{r}=-0.43$; salience-limbic: $p=0.006, \mathrm{r}=-0.33$; motor-auditory: $p=0.006$, $\mathrm{r}=0.32$; all FDR corrected], in line with the higher frontoparietal-limbic and salience-limbic connectivity in sadness, and the higher motor-auditory connectivity in amusement.

\section{Discussion}

We demonstrated that emotional states, specifically sadness and amusement, enhance the within- and between-subject similarity in the brain's functional connectome. Furthermore, by using a network science approach, we showed that the segregation and integration of brain network communities are linked to the subjective experience of emotional valence and reconfigure according to the emotional state. Specifically, we found that sadness was associated with higher modular integration relative to amusement and increased connectivity of cognitive control networks: the salience and fronto-parietal networks.

The emotional condition increased the within- and between-subject similarity in the functional connectome, in accordance with our hypotheses. This effect was found above and beyond the effect of movie watching. First, an individual's functional connectome was more similar between two emotional states (sadness vs. amusement) than between an emotional state and a neutral movie state. Second, participants were more similar to one another during emotional states than during the neutral movie state. Moreover, sadness was associated with higher between-subject similarity relative to amusement, in both the functional connectome and community structure.

The higher similarity between individuals during sadness in the functional connectome and community structure was not explained by increased engagement or interest, as higher interest was reported during the induction of amusement. In addition, the level of arousal did not differ between emotional states. Moreover, in the behavioral reports of emotion, higher between-subject similarity was found during amusement relative to sadness, and not vice versa. Thus, the similarity in the functional connectome did 
not merely reflect the similarity in the experience of emotion or could be attributed to general effects of engagement or arousal.

In the brain's topological organization, sadness was characterized by increased modular integration compared to amusement, measured by a greater diversity of functional connections across modules. Furthermore, the two emotions differed in between-module connectivity primarily of the fronto-parietal and salience networks. Both networks are implicated in task-related activity and cognitive control ${ }^{40}$. The salience network is centered on the anterior insula and anterior cingulate cortex and integrates external stimuli with internal states ${ }^{41}$. It was shown to mediate the interactions between externally oriented networks, such as the fronto-parietal, and internally oriented networks, particularly the default mode network ${ }^{42}$. The fronto-parietal network includes the dorsolateral prefrontal cortex and the posterior parietal cortex and was associated with working memory and goal-directed behavior ${ }^{41}$.

The connections of the fronto-parietal and salience modules, as well as the connectivity between these modules, were stronger in sadness. In addition, the salience module was more segregated in sadness, whereas the fronto-parietal module was more segregated in amusement. Taken together, we found that in sadness compared to amusement, the salience network was both more segregated, i.e., strongly intraconnected, and more integrated, i.e., diversely inter-connected. These metrics are not mutually exclusive, i.e., increased modular segregation does not necessarily imply isolation of the functional community. For example, a previous study showed that modular segregation and global integration increased simultaneously due to the strengthening of specific "hub" connections ${ }^{43}$.

The salience network emerged as a separate module only in sadness and amusement. In possible congruence, Wager et al. ${ }^{6}$ demonstrated that sadness and amusement were grouped together according to their preferential activity within the salience network. The fronto-parietal network, on the other hand, was identified in all four mental states (including the non-emotional ones). The fronto-parietal network was previously suggested to be most variable across tasks and suggested to flexibly interact with other networks to implement task demands ${ }^{8}$ 
Our results resonate with several theoretical accounts of emotion which emphasize a system-level approach ${ }^{6,44}$. In general agreement with those theories, we showed that each emotional state can be described by a whole-brain topological structure that involves all functional brain systems rather than a single system or brain structure. Adding to those theoretical foundations, we showed that differences between emotional states can be found in the segregation and integration attributes of functional brain communities/modules.

Importantly, at the individual level, we showed that the segregation and integration of brain modules are related to the behavioral report of emotional valence. The results were consistent with the direction of group-differences between the emotions in their modular organization. For example, if a brain module was more segregated in amusement relative to sadness at the group-level, its system segregation metric was positively associated with the emotional valence at the individual-level. This brain-behavior correspondence is crucial and demonstrates that the community structure captures important aspects of the subjective emotional experience. These findings may further aid in clinical and behavioral prediction. Behavioral prediction was argued to be more accurate when brain measures are taken from a relevant cognitive state ${ }^{45}$. Thus, characterizing the brain's community structure during naturalistic states of sadness and amusement (and other emotional states such as fear, for example) may enhance our ability to predict prospective psychiatric symptoms in at-risk and clinical populations.

Movie watching increased the within- and between-subject similarity in the functional connectome relative to rest. This is consistent with previous literature showing that movies increase synchronization between cortical signal fluctuations across individuals ${ }^{46,47}$. Studies that quantitatively compared functional connectivity between different movies are scarce, and initial findings indicate greater within-subject similarity during movies compared to rest, without differences between movies ${ }^{48,49}$. Notably, most studies that examined task effects on individual differences in the functional connectome did not include naturalistic stimuli ${ }^{32,50}$.

Furthermore, we provided a quantitative characterization of differences in modular organization between movie states and rest. We found that movie-watching, compared to rest, was characterized by 
higher modular segregation and higher modular integration, i.e., diversity of connections across modules. This is in agreement with a recent study ${ }^{51}$ which indicated reorganization of the community structure during movie watching relative to rest, however, it did not quantify the modular segregation or integration of the states.

Women were more similar to other women than men to other men in the brain's functional connectome. The magnitude of sex differences varied according to the mental state and the brain parcellation atlas, with amusement showing consistent higher similarity among women across brain atlases. The greater similarity among women is consistent with recent findings ${ }^{11}$. Moreover, out of the seven tasks of the HCP, the researchers found the largest sex differences during an emotion perception task. In a related study, Green et al. ${ }^{45}$ showed that the task that increases the ability to predict fluid intelligence from functional connectomes differs by sex. Interestingly, they showed that an emotion identification task was the best for predicting intelligence in women, whereas a working-memory task was the best for men.

We further found a higher similarity among women in the behavioral reports of emotion. In addition, amusement yielded the highest similarity among participants, compared to sadness or to the neutral state. Emotion similarity (i.e., in the subjective experience) among individuals is known to be associated with many interpersonal advantages, such as greater satisfaction, empathy, cooperation, and reduced stress ${ }^{52-54}$. Our results suggest that similarity in emotion, and thus its positive social effects, are more likely to be achieved during states of amusement and among women.

The brain parcellation atlas was shown to affect primarily the between-subject similarity and not the within-subject similarity. We found that the similarity between individuals was reduced with the use of smaller parcels. Smaller parcels are more sensitive to anatomical variability and imperfect coregistration, but also to functional variability, namely, slightly different anatomical locations for the same function across subjects. Salehi et al. ${ }^{55}$ showed recently that brain subdivisions based on functional connectivity data differ according to the task during which the data was acquired, even within an individual. In other words, parcels that were defined based on resting-state data, may not capture accurate functional units during other tasks or mental states. This may also explain the observation that among the four atlases tested, 
results obtained with the Shen atlas were the least consistent with those of the other atlases. While both Shen and Schaefer atlases were based on functional connectivity resting-state data, only the Schaefer atlas integrated local and global parcellation techniques ${ }^{31}$.

\section{Limitations}

Emotional states were elicited by movies, using different movie clips for each state (sadness, amusement, neutral). Consequently, low-level physical properties of the stimuli may possibly contribute to some of the differences between conditions in brain organization. This limitation cannot be entirely ruled out. However, to partially overcome this caveat, each emotional state was elicited by four movie clips, thus the statespecific functional connectomes captured the average interactions between brain regions across the different movies that induced a certain state. Characterizing states by averaging over several different movies instead of one was chosen to increase the generalizability of the findings, in addition to allowing for a continuous, naturalistic, and intense emotional experience. On a further note, low-level visual or auditory movie features were not associated with functional connectivity-based behavior prediction from movie-watching fMRI data ${ }^{20}$.

The study design does not enable us to conclude whether the findings are specific to the emotional categories (sadness, amusement) or related to emotional valence (negative, positive). Dimensional approaches of emotion characterize emotions based on a limited number of axes, such as valence, arousal, and approach-avoidance ${ }^{56,57}$. However, recent studies did not provide strong support for traditional dimensional approaches of emotion at the level of the brain's network organization ${ }^{5,6}$. Specifically, emotional categories were not indicated to be grouped according to their valence. For example, sadness and amusement, despite their contrasting valence, were more similar to one another in their brain representations than emotions of the same valence, such as sadness and fear ${ }^{6}$. In another study, neural activity patterns were most separable during the experience of distinct emotional categories and not 
differentiable according to valence and arousal ${ }^{5}$. Studies examining the functional connectome similarity and modular organization of additional emotional categories are needed.

\section{Conclusions}

By applying network science methods on the neural representations of intense naturalistic emotional states, this study deepened our understanding of the brain basis of emotion in humans. A modular organization of the brain during experiences of sadness and amusement was described, and the results extend previous attests for the essential importance of the brain's network segregation and integration, to the field of emotion. Our results suggest that the experience of emotion is linked to a reconfiguration of distributed, not emotion-specific, functional brain networks. The interaction patterns between functional networks, and not the networks themselves, are postulated to be the basis for an emotional state.

\section{CRediT authors statement}

Rotem Dan: Conceptualization; Data curation; Formal analysis; Investigation; Project administration; Resources; Visualization; Writing - Original draft; Writing - Review \& Editing. Marta Weinstock: Resources; Supervision; Writing - Review \& Editing. Gadi Goelman: Resources; Supervision; Writing Review \& Editing.

\section{Funding}

This work was supported by Dr. Marta Weinstock's funds from Drug Royalties.

\section{Declaration of competing interest}

The authors declare no conflict of interest. 


\section{References}

1. Ekman, P. Basic emotions. in Handbook of Cognition and Emotion 45-60 (Chichester, England, 1999).

2. Panksepp, J. Affective neuroscience: The foundations of human and animal emotions. (Oxford University Press, 2004).

3. Lindquist, K. A., Wager, T. D., Kober, H., Bliss-Moreau, E. \& Barrett, L. F. The brain basis of emotion: A meta-analytic review. Behav. Brain Sci. 35, 121-143 (2012).

4. Pessoa, L. A Network Model of the Emotional Brain. Trends in Cognitive Sciences vol. 21 357371 (2017).

5. Kragel, P. A. \& LaBar, K. S. Multivariate neural biomarkers of emotional states are categorically distinct. Soc. Cogn. Affect. Neurosci. 10, 1437-1448 (2015).

6. Wager, T. D. et al. A Bayesian Model of Category-Specific Emotional Brain Responses. PLoS Comput. Biol. 11, e1004066 (2015).

7. Barrett, L. F. \& Satpute, A. B. Large-scale brain networks in affective and social neuroscience: Towards an integrative functional architecture of the brain. Current Opinion in Neurobiology vol. 23 361-372 (2013).

8. Cole, M. W. et al. Multi-task connectivity reveals flexible hubs for adaptive task control. Nat. Neurosci. 16, 1348-1355 (2013).

9. Geerligs, L., Rubinov, M. \& Henson, R. N. State and trait components of functional connectivity: Individual differences vary with mental state. J. Neurosci. 35, 13949-13961 (2015).

10. Van Essen, D. C. \& Ugurbil, K. The future of the human connectome. NeuroImage vol. 62 12991310 (2012).

11. Finn, E. S. et al. Can brain state be manipulated to emphasize individual differences in functional connectivity? NeuroImage vol. 160 140-151 (2017).

12. Meunier, D., Lambiotte, R. \& Bullmore, E. T. Modular and hierarchically modular organization of brain networks. Frontiers in Neuroscience vol. 4200 (2010). 
13. Medaglia, J. D., Lynall, M. E. \& Bassett, D. S. Cognitive network neuroscience. J. Cogn.

Neurosci. 27, 1471-1491 (2015).

14. Cohen, J. R. \& D’Esposito, M. The Segregation and Integration of Distinct Brain Networks and Their Relationship to Cognition. J. Neurosci. 36, 12083-12094 (2016).

15. Riecher-Rössler, A. Sex and gender differences in mental disorders. The Lancet Psychiatry vol. 4 $8-9$ (2017).

16. Fu, C. H. Y. et al. Pattern Classification of Sad Facial Processing: Toward the Development of Neurobiological Markers in Depression. Biol. Psychiatry 63, 656-662 (2008).

17. Joormann, J., Siemer, M. \& Gotlib, I. H. Mood Regulation in Depression: Differential Effects of Distraction and Recall of Happy Memories on Sad Mood. J. Abnorm. Psychol. 116, 484-490 (2007).

18. Gruber, J., Hay, A. C. \& Gross, J. J. Rethinking emotion: Cognitive reappraisal is an effective positive and negative emotion regulation strategy in bipolar disorder. Emotion 14, 388-396 (2014).

19. Nastase, S. A., Goldstein, A. \& Hasson, U. Keep it real: rethinking the primacy of experimental control in cognitive neuroscience. Neuroimage 222, (2020).

20. Finn, E. S. \& Bandettini, P. A. Movie-watching outperforms rest for functional connectivity-based prediction of behavior. Neuroimage 235, (2021).

21. Vanderwal, T., Eilbott, J. \& Castellanos, F. X. Movies in the magnet: Naturalistic paradigms in developmental functional neuroimaging. Developmental Cognitive Neuroscience vol. 36100600 (2019).

22. Rottenberg, J., Ray, R. \& Gross, J. Emotion elicitation using films. in handbook of emotion elicitation and assessment (ed. A. Coan and JJB Allen) (2007).

23. Schaefer, A., Nils, F., Sanchez, X. \& Philippot, P. Assessing the effectiveness of a large database of emotion-eliciting films: A new tool for emotion researchers. Cogn. Emot. 24, 1153-1172 (2010). 
24. Farb, N. A. S. et al. Minding one's emotions: mindfulness training alters the neural expression of sadness. Emotion 10, 25-33 (2010).

25. Gross, J. J. \& Levenson, R. W. Emotion elicitation using films. Cogn. Emot. 9, 87-108 (1995).

26. Dan, R. et al. Sex differences during emotion processing are dependent on the menstrual cycle phase. Psychoneuroendocrinology 100, 85-95 (2019).

27. Whitfield-Gabrieli, S. \& Nieto-Castanon, A. Conn: a functional connectivity toolbox for correlated and anticorrelated brain networks. Brain Connect. 2, 125-41 (2012).

28. Tzourio-Mazoyer, N. et al. Automated anatomical labeling of activations in SPM using a macroscopic anatomical parcellation of the MNI MRI single-subject brain. Neuroimage 15, 27389 (2002).

29. Desikan, R. S. et al. An automated labeling system for subdividing the human cerebral cortex on MRI scans into gyral based regions of interest. Neuroimage 31, 968-980 (2006).

30. Shen, X., Tokoglu, F., Papademetris, X. \& Constable, R. T. Groupwise whole-brain parcellation from resting-state fMRI data for network node identification. Neuroimage 82, 403-415 (2013).

31. Schaefer, A. et al. Local-global parcellation of the human cerebral cortex from intrinsic functional connectivity mri. Cereb. Cortex 28, 3095-3114 (2018).

32. Cole, M. W., Bassett, D. S., Power, J. D., Braver, T. S. \& Petersen, S. E. Intrinsic and task-evoked network architectures of the human brain. Neuron 83, 238-251 (2014).

33. Lancichinetti, A. \& Fortunato, S. Consensus clustering in complex networks. Sci. Rep. 2, 1-7 (2012).

34. Rubinov, M. \& Sporns, O. Complex network measures of brain connectivity: Uses and interpretations. Neuroimage 52, 1059-1069 (2010).

35. Reichardt, J. \& Bornholdt, S. Statistical mechanics of community detection. Phys. Rev. E - Stat. Nonlinear, Soft Matter Phys. 74, 016110 (2006).

36. Rubinov, M. \& Sporns, O. Weight-conserving characterization of complex functional brain networks. Neuroimage 56, 2068-2079 (2011). 
37. Kuncheva, L. I. \& Hadjitodorov, S. T. Using diversity in cluster ensembles. in Conference Proceedings - IEEE International Conference on Systems, Man and Cybernetics vol. 2 1214-1219 (2004).

38. Chan, M. Y., Park, D. C., Savalia, N. K., Petersen, S. E. \& Wig, G. S. Decreased segregation of brain systems across the healthy adult lifespan. Proc. Natl. Acad. Sci. U. S. A. 111, E4997-E5006 (2014).

39. Guimerà, R. \& Amaral, L. A. N. Cartography of complex networks: Modules and universal roles. J. Stat. Mech. Theory Exp. 2005, 1-13 (2005).

40. Power, J. D. \& Petersen, S. E. Control-related systems in the human brain. Current Opinion in Neurobiology vol. 23 223-228 (2013).

41. Seeley, W. W. et al. Dissociable Intrinsic Connectivity Networks for Salience Processing and Executive Control. J. Neurosci. 27, 2349-2356 (2007).

42. Sridharan, D., Levitin, D. J. \& Menon, V. A critical role for the right fronto-insular cortex in switching between central-executive and default-mode networks. Proc. Natl. Acad. Sci. U. S. A. 105, 12569-12574 (2008).

43. Baum, G. L. et al. Modular Segregation of Structural Brain Networks Supports the Development of Executive Function in Youth. Curr. Biol. 27, 1561-1572.e8 (2017).

44. Pessoa, L. Embracing integration and complexity: placing emotion within a science of brain and behaviour. Cogn. Emot. 33, 55-60 (2019).

45. Greene, A. S., Gao, S., Scheinost, D. \& Constable, R. T. Task-induced brain state manipulation improves prediction of individual traits. Nat. Commun. 9, (2018).

46. Lankinen, K., Saari, J., Hari, R. \& Koskinen, M. Intersubject consistency of cortical MEG signals during movie viewing. Neuroimage 92, 217-224 (2014).

47. Hasson, U., Nir, Y., Levy, I., Fuhrmann, G. \& Malach, R. Intersubject Synchronization of Cortical Activity during Natural Vision. Science (80-. ). 303, 1634-1640 (2004).

48. Vanderwal, T. et al. Individual differences in functional connectivity during naturalistic viewing 
conditions. Neuroimage 157, 521-530 (2017).

49. Tian, L., Ye, M., Chen, C., Cao, X. \& Shen, T. Consistency of functional connectivity across different movies. Neuroimage 233, 117926 (2021).

50. Gratton, C. et al. Functional Brain Networks Are Dominated by Stable Group and Individual Factors, Not Cognitive or Daily Variation. Neuron 98, 439-452.e5 (2018).

51. Kim, D., Kay, K., Shulman, G. L. \& Corbetta, M. A new modular brain organization of the bold signal during natural vision. Cereb. Cortex 28, 3065-3081 (2018).

52. Townsend, S. S. M., Kim, H. S. \& Mesquita, B. Are You Feeling What I'm Feeling? Emotional Similarity Buffers Stress. Soc. Psychol. Personal. Sci. 5, 526-533 (2014).

53. Barsade, S. G. The ripple effect: Emotional contagion and its influence on group behavior. Administrative Science Quarterly vol. 47644 (2002).

54. Locke, K. D. \& Horowitz, L. M. Satisfaction in Interpersonal Interactions as a Function of Similarity in Level of Dysphoria. J. Pers. Soc. Psychol. 58, 823-831 (1990).

55. Salehi, M. et al. There is no single functional atlas even for a single individual: Functional parcel definitions change with task. Neuroimage 208, 116366 (2020).

56. Russell, J. A. A circumplex model of affect. J. Pers. Soc. Psychol. 39, 1161-1178 (1980).

57. Maxwell, J. S. \& Davidson, R. J. Emotion as motion: Asymmetries in approach and avoidant actions. Psychol. Sci. 18, 1113-1119 (2007).

58. Fruchterman, T. M. J. \& Reingold, E. M. Graph drawing by force-directed placement. Softw. Pract. Exp. 21, 1129-1164 (1991).

59. Xia, M., Wang, J. \& He, Y. BrainNet Viewer: a network visualization tool for human brain connectomics. PLoS One 8, e68910 (2013).

60. Batagelj, V. \& Mrvar, A. Pajek-Program for Large Network Analysis. Connections 21, 47-57 (1998). 


\section{Figure legends}
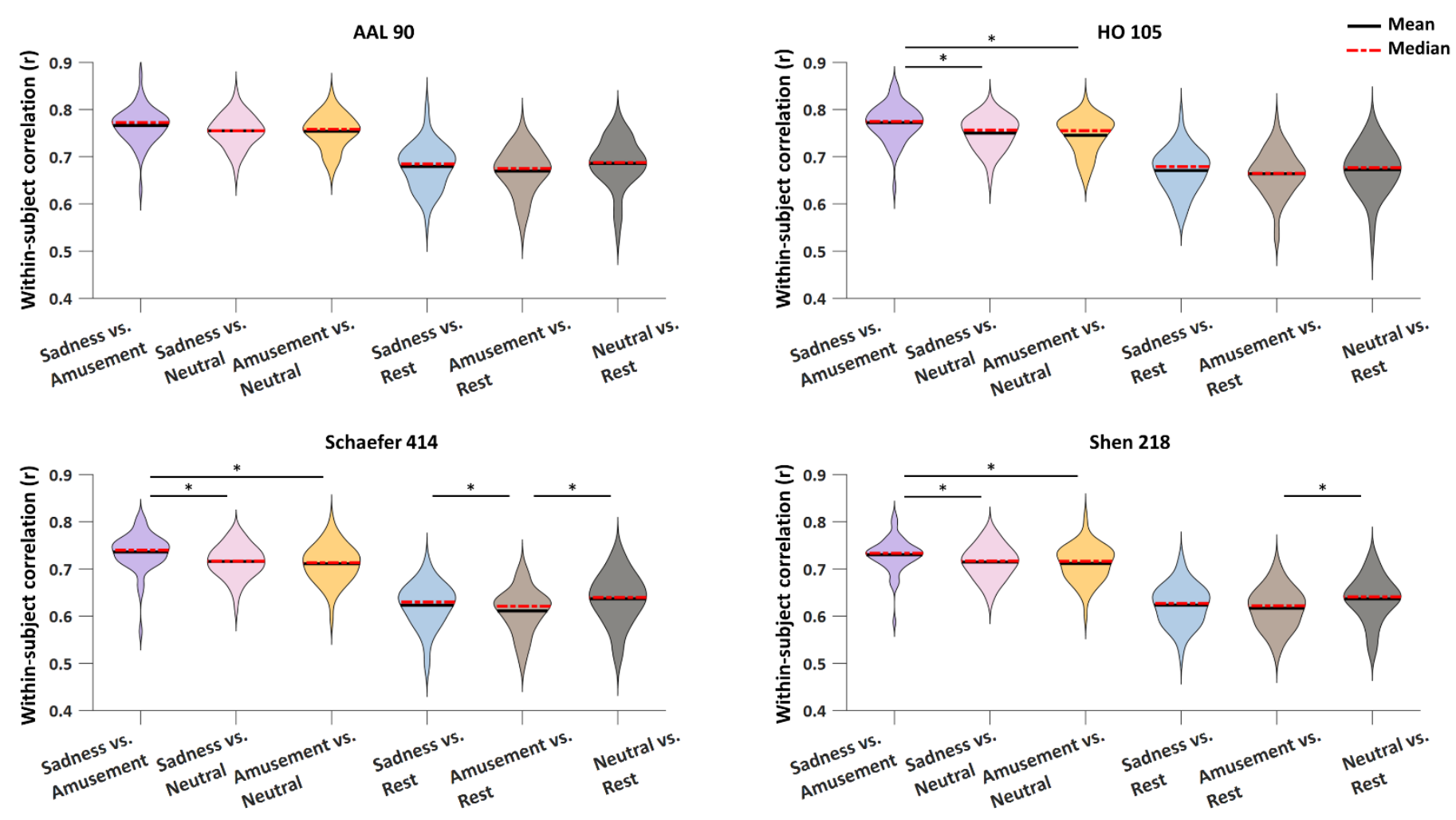

${ }^{*} p<0.05$ Sidak corrected. Also significant: all pairwise differences between two movie states ( 3 most left violins) and a movie and resting state ( 3 most right violins).

Figure 1. Within-subject similarity in the functional connectome is dependent on the brain state. The within-subject similarity in the functional connectome (Pearson's correlation $r$ values) is presented as a function of the pair of brain states, for the four parcellation atlases. An individual's functional connectome was more similar between two movie states (three most left violins) than between a movie and rest (three most right violins) (pairwise differences: Cohen's $d=1.35-3.08)$. Furthermore, the functional connectome was more similar between two emotional states (sadness vs. amusement) than between an emotional state and the neutral movie state (Cohen's $d=0.43-0.81$ ), for all atlases except the AAL. In each violin, the median is indicated by dashed red lines and the mean by solid black lines. Significant differences are marked by asterisks $(*), p<0.05$ Sidak corrected. 
(a)
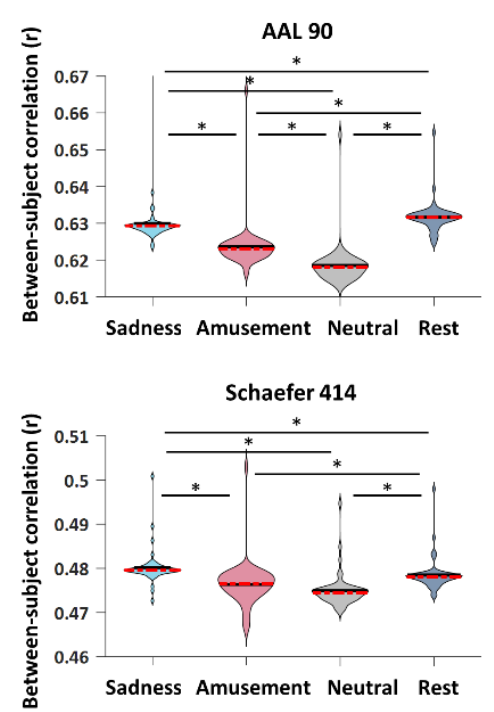

${ }^{*} p<0.05$ Sidak corrected

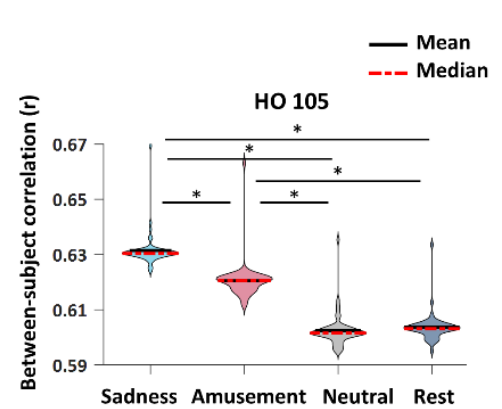

(b) Women-to-women
$\bigcirc$ Men-to-men
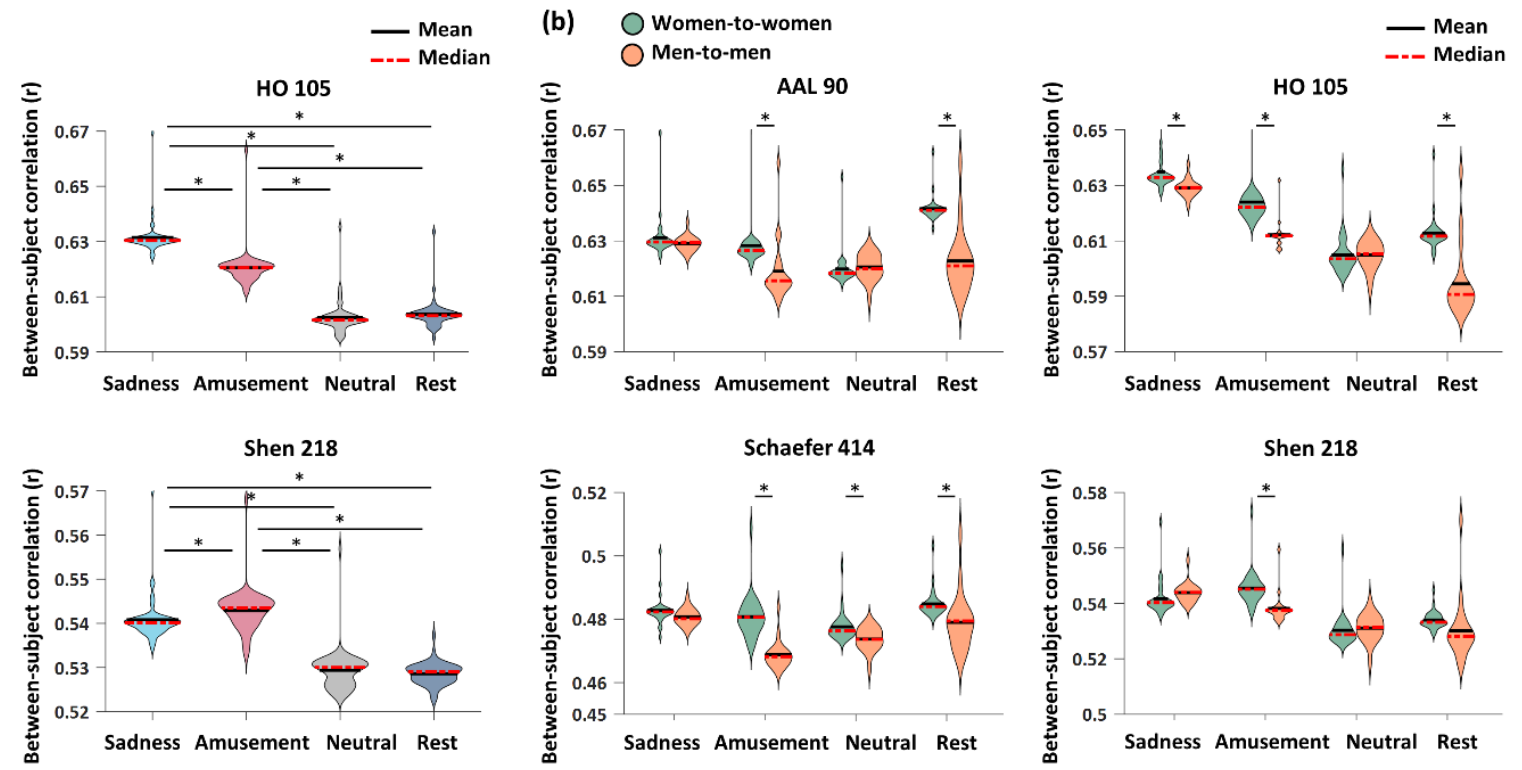

Sadness Amusement Neutral Rest

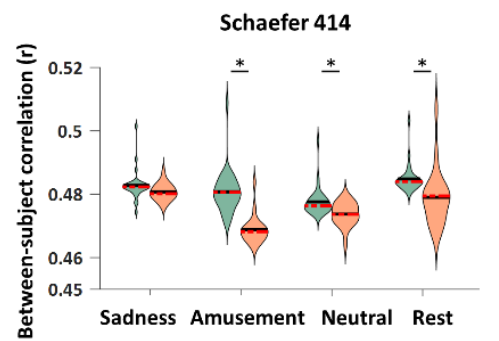

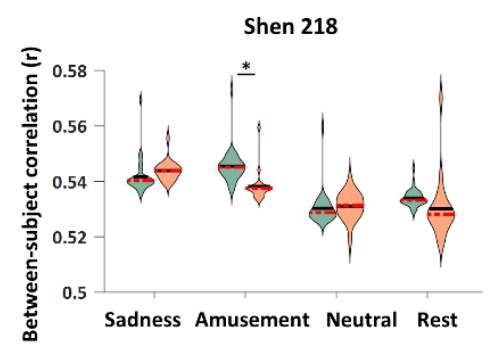

Figure 2. Between-subject similarity in the functional connectome is dependent on the brain state and

sex. The between-subject similarity in the functional connectome (Pearson's correlation $r$ values) is presented as a function of the brain state, for the four parcellation atlases. (a) Individuals were more similar to one another during emotional states (sadness, amusement) than during the neutral movie state, regardless of the parcellation atlas (Cohen's $d=1.79-9.55)$. Higher between-subject similarity was found during sadness relative to amusement (Cohen's $d=1.06-3.62$ ), except for the Shen atlas. (b) The effect of sex was examined by computing women-to-women (green) and men-to-men (orange) similarity. Women were more similar to other women than men to other men, across states, for all atlases except for the Shen atlas. Amusement showed significant sex differences across all atlases (Cohen's $d=0.44-0.96)$. In each violin, the median is indicated by dashed red lines and the mean by solid black lines. Significant differences are marked by asterisks $(*), p<0.05$ Sidak corrected. 
(a)

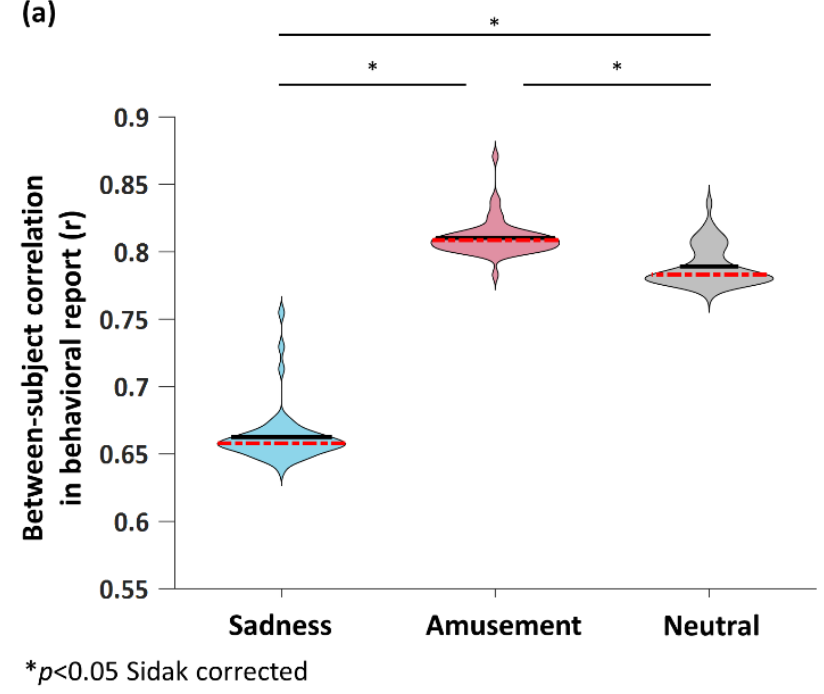

(b)

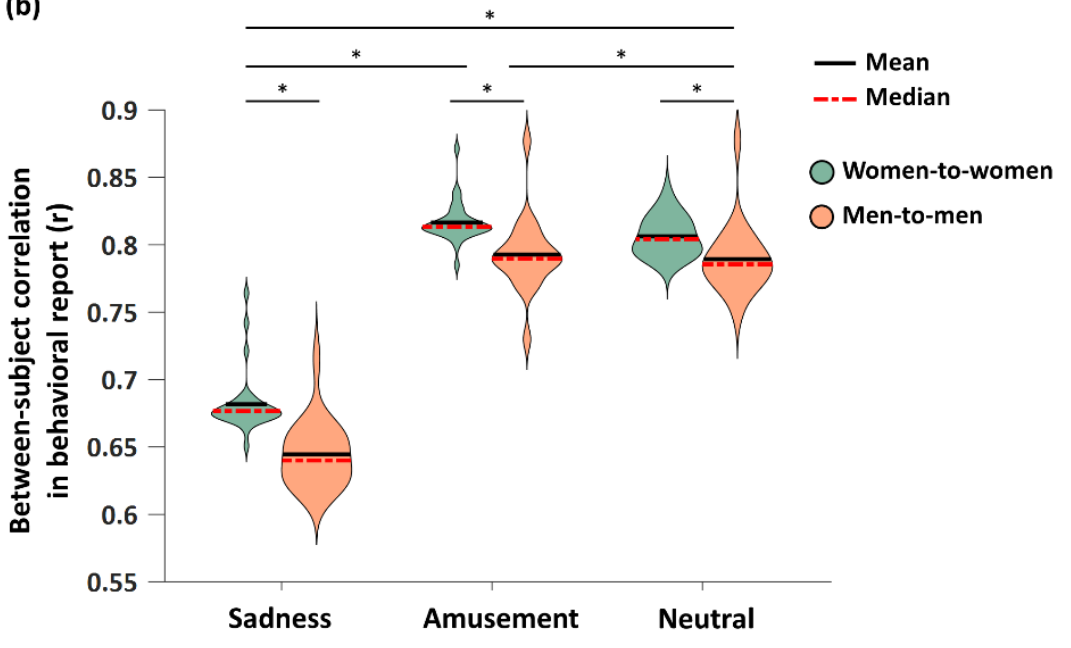

Figure 3. Higher similarity in the behavioral report of emotional experience during amusement and

among women. (a) The between-subject similarity in the subjective report of emotional experience is presented as a function of the emotional state. Sadness is indicated in blue, amusement in red, and neutral in gray. Higher similarity in the reported emotional experience was found during amusement relative to sadness or neutral (pairwise state differences: Cohen's $d=2.01-9.49)$. (b) Women were more similar to other women than men to other men, regardless of the emotional state (pairwise sex differences: Cohen's $d=0.35$ 0.74). In each violin, the median is indicated by dashed red lines and the mean by solid black lines. Significant differences are marked by asterisks $(*), p<0.05$ Sidak corrected. 
Sadness

(a) Brain space

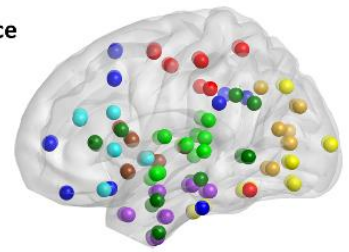

Amusement

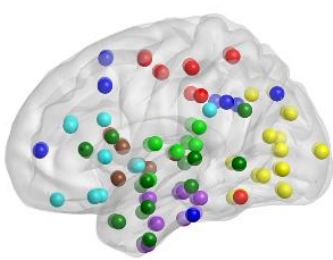

Neutral

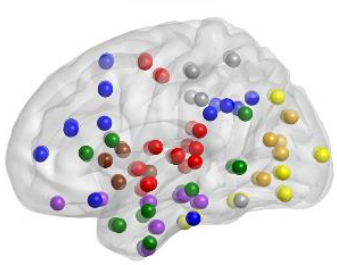

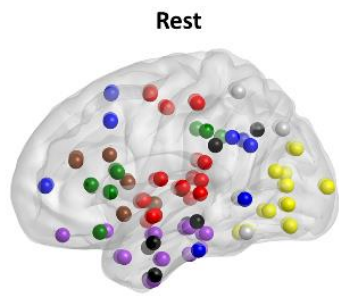

(b) Force-directed

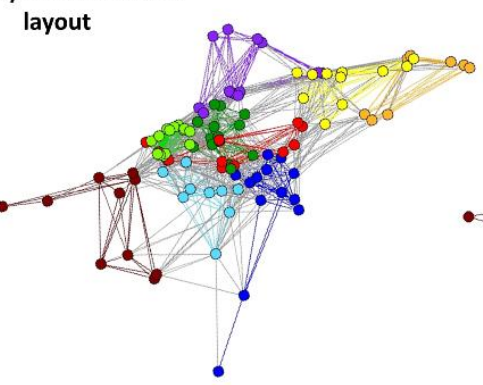

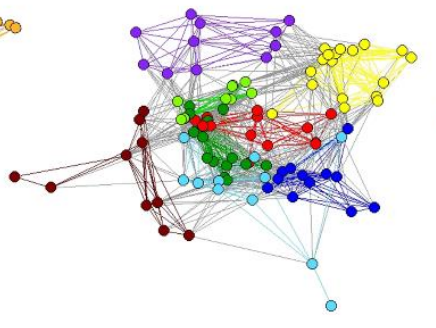

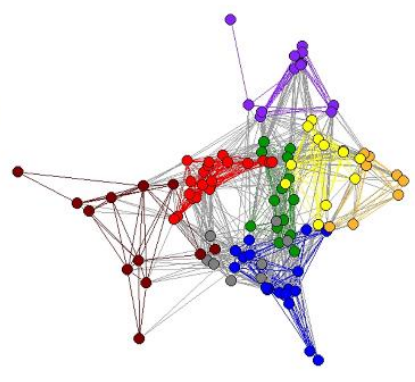

Fronto-parietal

OSalience

Language

Posterior sensory

Temporal-pDMN

Sensorimotor

Auditory

Visual primary

$\bigcirc$ Visual secondary

Limbic

Basal ganglia

Figure 4. Network communities for different brain states. Communities (i.e., modules) identified for sadness, amusement, neutral, and rest are denoted by colors. (a) Anatomical brain space representation of the network communities: brain regions are indicated by circles. (b) Force-directed layouts of the network communities are presented using the Fruchterman-Reingold algorithm ${ }^{58}$. In this layout, connections act as spring-like attractive forces to position nodes in space such that nodes with more shared connections are pulled closer together. The community structure of each brain state was identified using group-level consensus clustering on the individual-level connectivity matrices. For visualization purposes only, the group-averaged functional connections are used here to represent the edges between the nodes in the graphs, and the graphs are displayed at a density of 0.2 , i.e., the top $20 \%$ of connections are shown for each brain state. Brain space layouts were visualized using BrainNet Viewer ${ }^{59}$ and force-directed layouts were visualized using Pajek ${ }^{60}$. pDMN, posterior default mode network. 
(a)

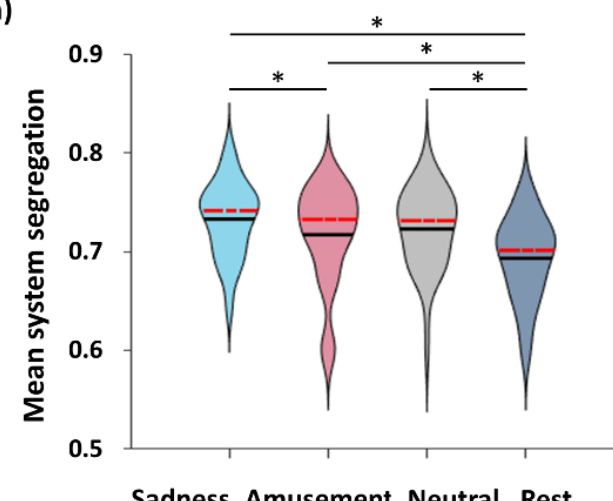

(c)

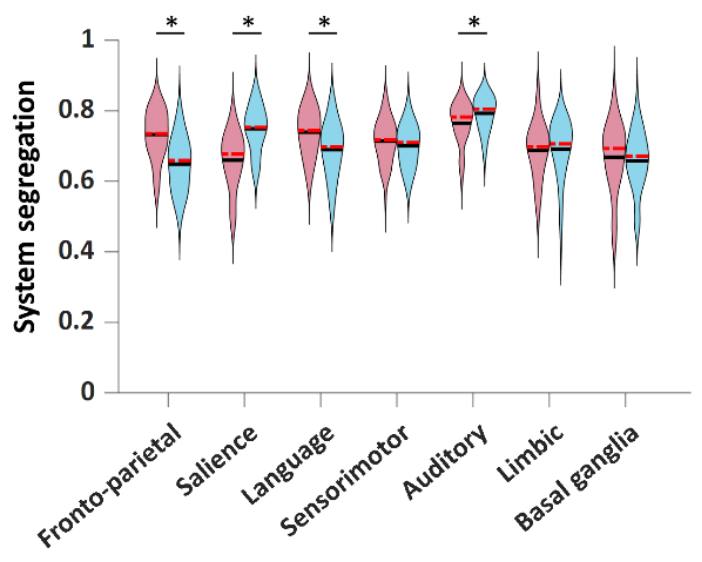

(b)

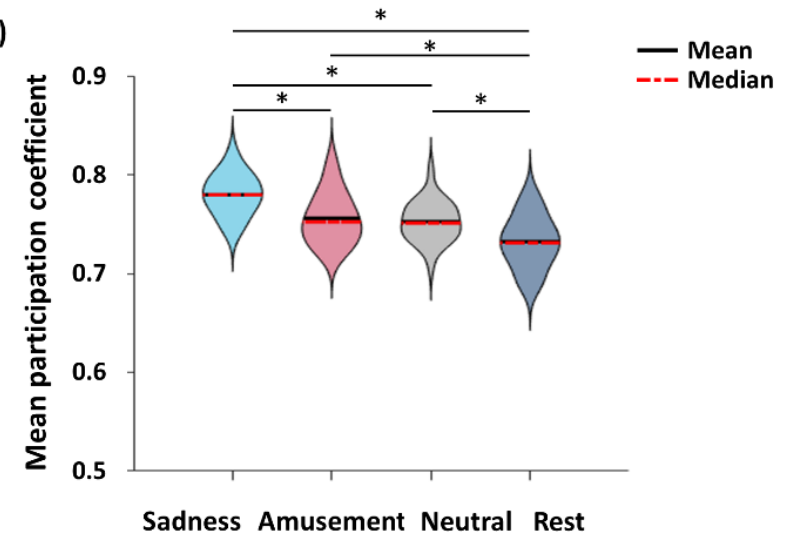

(d)

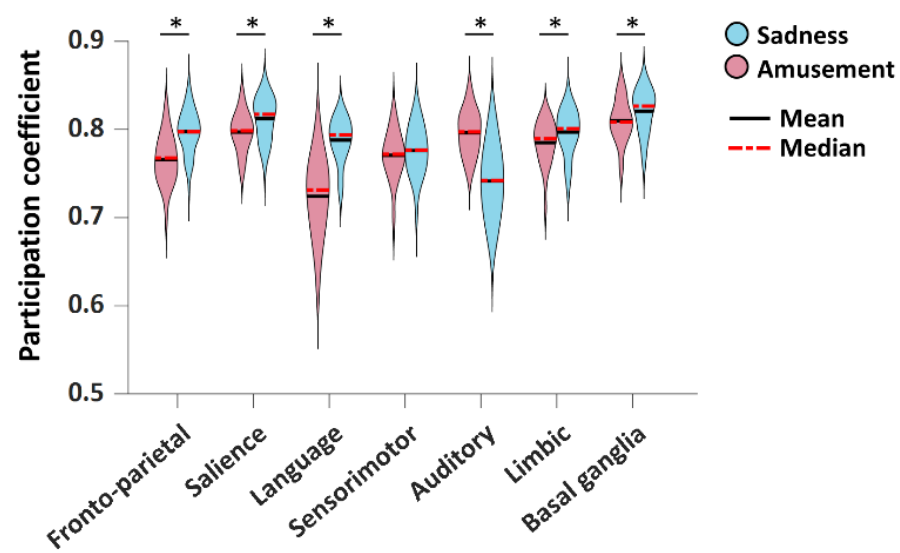

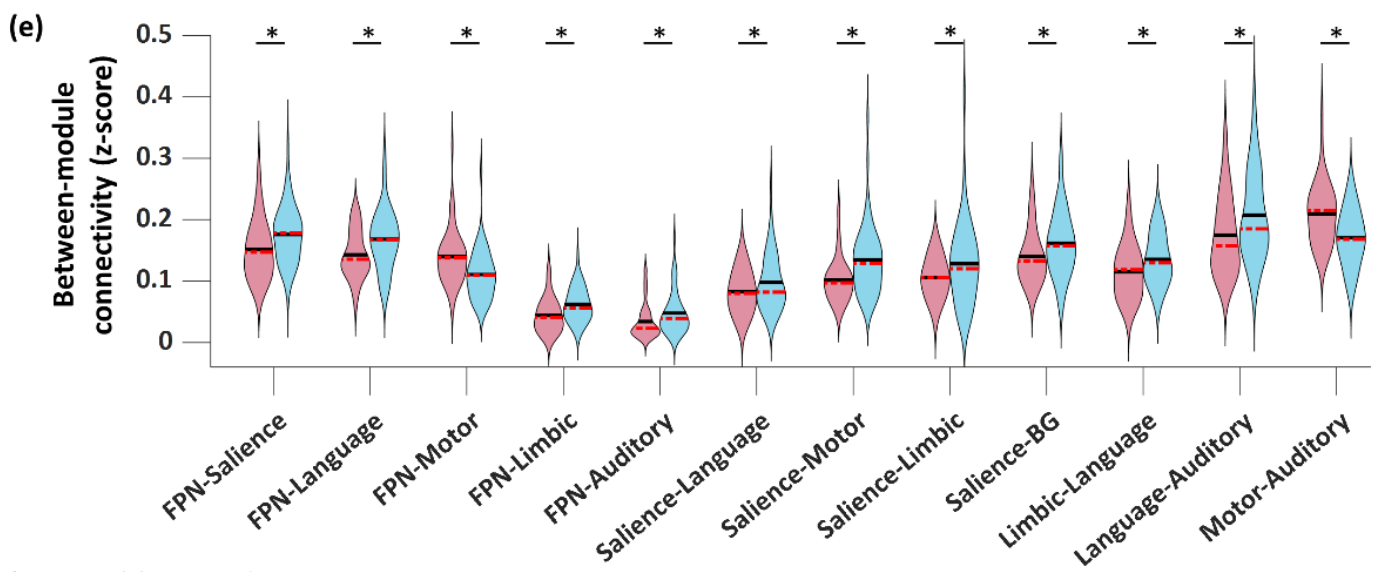

${ }^{*} p<0.05$ Sidak corrected

Figure 5. Modular segregation and integration of emotional brain states. (a) The mean system segregation (across all modules) is presented as a function of the mental state. Higher values indicate higher segregation. (b) The mean participation coefficient (across all nodes) is presented as a function of the mental state. Higher values indicate greater diversity of connections across modules, i.e., higher integration. For 
sadness (blue) and amusement (red), the (c) system segregation, (d) participation coefficient, and (e) pairwise between-module connectivity are presented as a function of the brain module. In each violin, the median is indicated by dashed red lines and the mean by solid black lines. Significant differences between emotional states are marked by asterisks $\left(^{*}\right), p<0.05$ Sidak corrected. BG, basal ganglia; FPN, frontoparietal network. 